\title{
A review of recent case reports of cenesthopathy in Japan
}

Tohru Takahashi, MD, PhD, ${ }^{1}$ Tomonori Fuke, $\mathrm{MD},{ }^{2}$ Shinsuke Washizuka, MD, $\mathrm{PhD},{ }^{1}$

Tokiji Hanihara, $\mathrm{MD}, \mathrm{PhD},{ }^{3}$ and Naoji Amano, $\mathrm{MD}, \mathrm{PhD}^{1}$

${ }^{1}$ Department of Psychiatry, Shinshu University School of Medicine, Nagano, Japan

${ }^{2}$ Komoro Plateau Hospital, Nagano, Japan

${ }^{3}$ School of Health Sciences, Shinshu University, Nagano, Japan

* Correspondence: Tohru Takahashi, MD, PhD, Department of Psychiatry, Shinshu

University School of Medicine, 3-1-1 Asahi, Matsumoto, Nagano 390-8621 Japan.

E-mail: takatoh@shinshu-u.ac.jp

Tel.: +81-263-37-2638; Fax: +81-263-36-1772

Number of figures: 2

Number of words in the manuscript: 1455 words

Number of words in the abstract: 72 words

Running title: A review of cenesthopathy in Japan 


\section{ABSTRACT}

Idiopathic abnormal bodily sensations (cenesthesic symptoms) are exhibited in a wide variety of mental illness. In Japan, patients with abnormal bodily sensations are often diagnosed with cenesthopathy. This study reviewed recent case reports of cenesthopathy. Of the 100 identified cases, young patients were more commonly males with predominant bodily cenesthesic symptoms, while older patients ( $\geq 40$ years) were more commonly females with cenesthesic symptoms restricted to the oral cavity (oral cenesthopathy).

Keywords: cenesthopathy, cenesthesia, delusional disorder, somatoform disorder 


\section{INTRODUCTION}

Cenesthopathy is a notion introduced by Dupré and Camus in 1907 to describe

pathologic bodily sensations as phenomenology in mental illness. ${ }^{1}$ According to the DSM-IV, ${ }^{2}$ classical "cenesthopathies" are classified as either delusional disorder, somatic type, or somatoform disorder. In the ICD-10, "cenesthopathic schizophrenia"4 is included, but is not further defined and falls within the category "other schizophrenia" (F20.8). In Japan, some classical useful psychiatric nosologies (e.g., cenesthopathy, late catatonia, and involutional melancholia) and the operational diagnostic criteria have been used as clinical entities in combination with operational diagnostic criteria.

Furthermore, "cenesthopathy" has traditionally been used not only as a description of pathologic bodily sensations, but also as a psychiatric diagnosis. Patients that predominantly manifest abnormal bodily sensations are often diagnosed with cenesthopathy and many cases in Japan are still reported under the concept of cenesthopathy. Monosymptomatic hypochondriasis psychosis (MHP) $)^{5-7}$ is a disorder characterized by a single hypochondriacal complaint supported by delusional beliefs. The 4 most common forms of MHP are: (1) delusion of body odor (olfactory reference syndrome), (2) dysmorphophobia, (3) delusional parasitosis, and (4) cenesthopathy. Delusion of body odor and dysmorphophobia usually begin in adolescence, while 
delusional parasitosis is frequently found in the elderly. ${ }^{8}$ In contrast, the age distribution for cenesthopathy is controversial. The purpose of this study was to evaluate the characteristics of cases recently reported as cenesthopathy.

\section{METHODS}

A literature review was conducted using both MEDLINE and the Japana Centra Revuo Medicina (JCRM), a medical database of Japanese papers, for cases reported as "cenesthopathy" between 2000 and 2010. Patient age, sex, and both symptoms and treatments for each case were evaluated.

\section{RESULTS}

In MEDLINE, 2 reports from Japan were identified. ${ }^{9,10}$ Watanabe et al. ${ }^{9}$ reported 23 consecutive cases in patients $\leq 30$ years of age (19 males; mean \pm SD age: $20.9 \pm 3.4$ years) in which the sites of cenesthesic symptoms varied (head, chest, abdomen, and limbs). No patients reported oral cenesthesic symptoms. Honma et al. ${ }^{10}$ reported the results of Rorschach tests in 28 patients with oral cenesthopathy ( 3 males; mean age: $55.1 \pm 14.5$ years).

From the JCRM database, 49 reports including 77 cases were collected (24 
males; age range: $24-81$ years; mean age: $55.6 \pm 13.1$ years). Cenesthesic symptoms were oral in 51 patients and bodily (non-oral) in 26 patients. Fifty-seven patients were diagnosed with primary cenesthopathy (18 males; mean age: $55.2 \pm 12.5$ years) and 40 of these had symptoms restricted to the oral cavity (non-oral: 17 patients). Psychiatric diagnoses (Axis I disorders) were made in 20 patients ( 6 males; mean age: $56.6 \pm 15.0$ years; oral: 11 patients; non-oral: 9 patients) patients with cenesthopathy and included mood disorders (10 patients), paranoid disorder (3), dementia (3), schizophrenia (2), and organic brain disorders (2). Response to treatment was reported for 39 patients, with 16 experiencing symptomatic improvement with atypical antipsychotics (aripiprazole [6], olanzapine [4], quetiapine [3], risperidone [1], perospirone [1], and blonanserin [1]), 10 with antidepressant therapy (milnacipran [6], fluvoxamine [3], clomipramine [1]), lithium carbonate (2), a combination of 2 classes of drugs (5), donepezil (1), and electroconvulsive therapy (5).

When all identified cases were considered together (23 reported by Watanabe et al. and 77 collected from the JCRM, Figure 1), non-oral symptoms were more common in younger patients ( $\leq 39$ years) and oral symptoms in older patients ( $\geq 40$ years). There were more male patients in the younger age group (male/female: $23 / 11$ ) and more female patients in the older age group (male/female: 20/46) (Figure 2). 


\section{DISCUSSION}

In concordance with previous studies, ${ }^{11,12}$ this study shows that cenesthopathy is more common in adolescent male patients, but that more middle-aged to elderly females report cenesthopathies. Furthermore, this study suggests that young patients more often experience bodily symptoms, while oral symptoms are frequent in patients $\geq$ 40 years of age.

In 1957, Huber ${ }^{13}$ proposed "cenesthetic schizophrenie" as a subtype of schizophrenia and defined the disorder as a schizophrenia in which bizarre, peculiar, abnormal bodily sensations dominate, are difficult-to-describe, and have a chronic, insidious course, with typical schizophrenic symptoms limited to brief psychotic episodes. Huber noted a close relationship between cenesthesic phenomenology and spontaneous sensations of the thalamus. ${ }^{13,14} \mathrm{He}$ also suggested that central brain atrophy, as identified in some patients by pneumoencephalography, computed tomography, and magnetic resonance imaging, might play an important role in developing abnormal bodily sensations. ${ }^{13,14}$ In Japan, studies on cenesthopathy began in earnest in the 1960s. Hozaki ${ }^{15}$ described 12 patients with abnormal bodily sensations ( 7 males; mean age: 28.0 years), some of which could be diagnosed with schizophrenia; however, many of the rest were difficult to classify into an established category. Yoshimatsu ${ }^{16}$ reported on 
30 patients (23 males; mean age: 30.1 years), half of whom could be diagnosed with schizophrenia. However, some of their patients had depressive psychosis. At that time, most reported cases were of young patients and the primary concern of these early studies was that the patients with cenesthetic symptoms were at least partially distinct from schizophrenia.

Data from the current review suggests that the incidence of female patients with oral cenesthesic symptoms at age $\geq 40$ years may be increasing. ${ }^{12}$ Axis I psychiatric diagnoses were made in only $20 \%$ and both mood disorders and organic brain disease were relatively frequent. Organic causes of oral cenesthesia are of concern, particularly in elderly patients. For example, one report illustrates a patient that developed oral cenesthesia and a parasitic delusion, resulting from direct damage to the thalamocortical tract, ${ }^{17}$ while another discusses a patient with stroke in whom cortical reorganization was involved in the development of oral cenesthesia. ${ }^{18}$ Finally, studies have shown that oral cenesthopathy often occurs after prolonged pain or after surgical treatment (e.g., tooth extraction). Sensitization of the somatosensory system or remodeling of the cortical sensory areas may be involved. The cortical somatosensory areas that map to the oral cavity are large ${ }^{19}$ and may be susceptible to the effects of aging. We propose that oral cenesthopathy of middle-aged to elderly patients should be distinguished from 
that of younger patients, since involvement of an organic condition should be suspected.

Future studies are necessary to clarify the exact neurologic basis of cenesthesic

phenomena, especially in patients with oral cenesthopathy.

\section{ACKNOWLEDGMENTS}

The authors declare no conflicts of interest. 


\section{REFERENCES}

1. Dupré E, Camus P. Les cénesthopathies. Encéphale 1907; 4: 616-631 (in French).

2. American Psychiatric Association. Diagnostic and Statistical Manual of Mental

Disorders, 4th edn. Washington, DC: American Psychiatric Association, 1994.

3. World Health Organization. The ICD-10 Classification of Mental and Behavioural

Disorders: Clinical Descriptions and Diagnostic Guidelines. Geneva: World Health

Organization, 1992.

4. Jenkins G, Röhricht F. From cenesthesias to cenesthopathic schizophrenia: a historical and phenomenological review. Psychopathology 2007; 40: 361-368.

5. Rinding J, Munro A. Pimozide in the treatment of monosymptomatic hypochondriacal psychosis. Acta Psychiatr Scand 1975; 52: 23-30.

6. Reilly TM. Monosymptomatic hypochondriacal psychosis; Presentation and treatment. Proc R Soc Med 1977; 70 (Suppl 10): 39-43.

7. Munro A. Monosymptomatic hypochondriacal psychosis. Br J Psychiatry Suppl 1988; 153 (Suppl 2): 37-40.

8. Trabert W. 100 years of delusional parasitosis. Meta-analysis of 1,223 case reports. Psychopathology 1995; 28: 238-246.

9. Watanabe H, Takahashi T, Tonoike T, Suwa M, Akahori K. Cenesthopathy in 
adolescence. Psychiatry Clin Neurosci 2003; 57: 23-30.

10. Honma F, Kimura M, Endo S, Ohtsu M, Okada T, Satoh T. Oral cenesthopathy examined by Rorschach test. Psychiatry Clin Neurosci 2006; 60: 154-159.

11. Takahashi T, Yoshimatsu K. A consideration of the clinical classification of cenesthopathia; from a viewpoint of case studies. Seishin Igaku 1998; 40: 507-516 (in Japanese).

12. Takahashi T, Tamaru T, Imai J et al. Pathology in senile patients with abnormal body sensation. Psychogeriatrics 2001; 1: 139-142.

13. Huber G. Die coenästhetische schizophrenie. Fortschr Neurol Psychiatr 1957; 25: 491-520 (in German).

14. Huber G. "Cenesthetic schizophrenia" - a subtype of schizophrenic disease. Neurology, Psychiatry and Brain Research 1992; 1: 54-60.

15. Hozaki H. Cenestopathia and related condition. Seishin Igaku Zasshi 1960; 2: 325-332 (in Japanese).

16. Yoshimatsu K. The psychopathology of cenesthopathia. Seishin Shinkeigaku Zasshi 1966; 68: 872-890 (in Japanese).

17. Hanihara T, Takahashi T, Washizuka S, Ogihara T, Kobayashi M. Delusion of oral parasitosis and thalamic pain syndrome. Psychosomatics 2009; 50: 534-537. 
18. Kato Y, Muramatsu T, Kato M, Shibukawa Y, Shintani M, Yoshino F. Cortical reorganization somatic delusional psychosis: A magnetoencephalographic study. Psychiatry Res 2006; 146: 91-95.

19. Penfield WG, Boldrey E. Somatic motor and sensory representation in the cerebral cortex of man as studied by electrical stimulation. Brain 1937; 60, 389-443. 


\section{FIGURE LEGENDS}

Figure 1. Frequency distribution of 100 reported cenesthopathy cases. Black bars $=$ patients with non-oral cenesthopathy. White bars = patients with oral cenesthopathy.

Figure 2. Male to female ratio of 100 reported cenesthopathy cases. Black bars $=$ male patients. White bars $=$ female patients. 
Figure 1

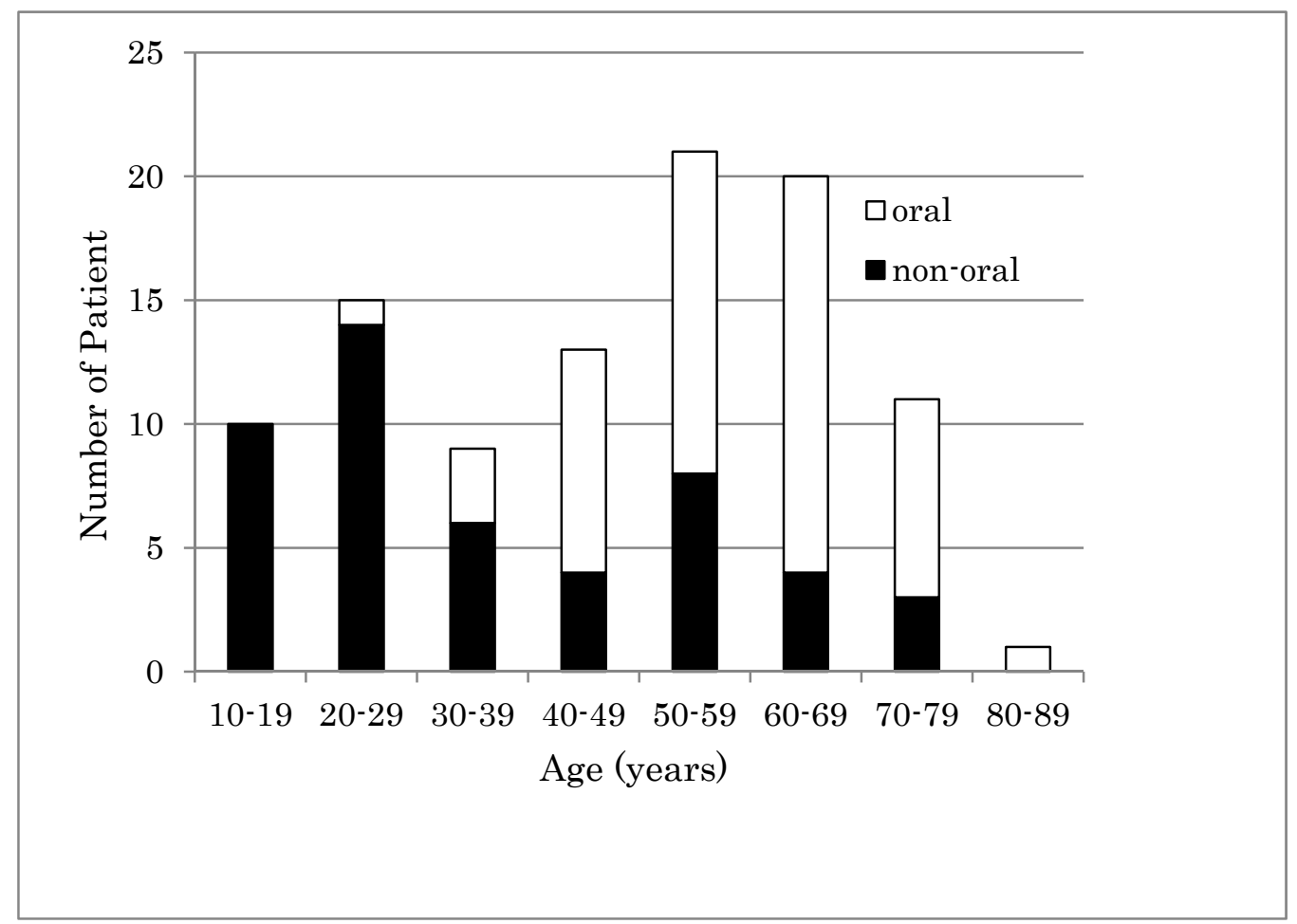


Figure 2

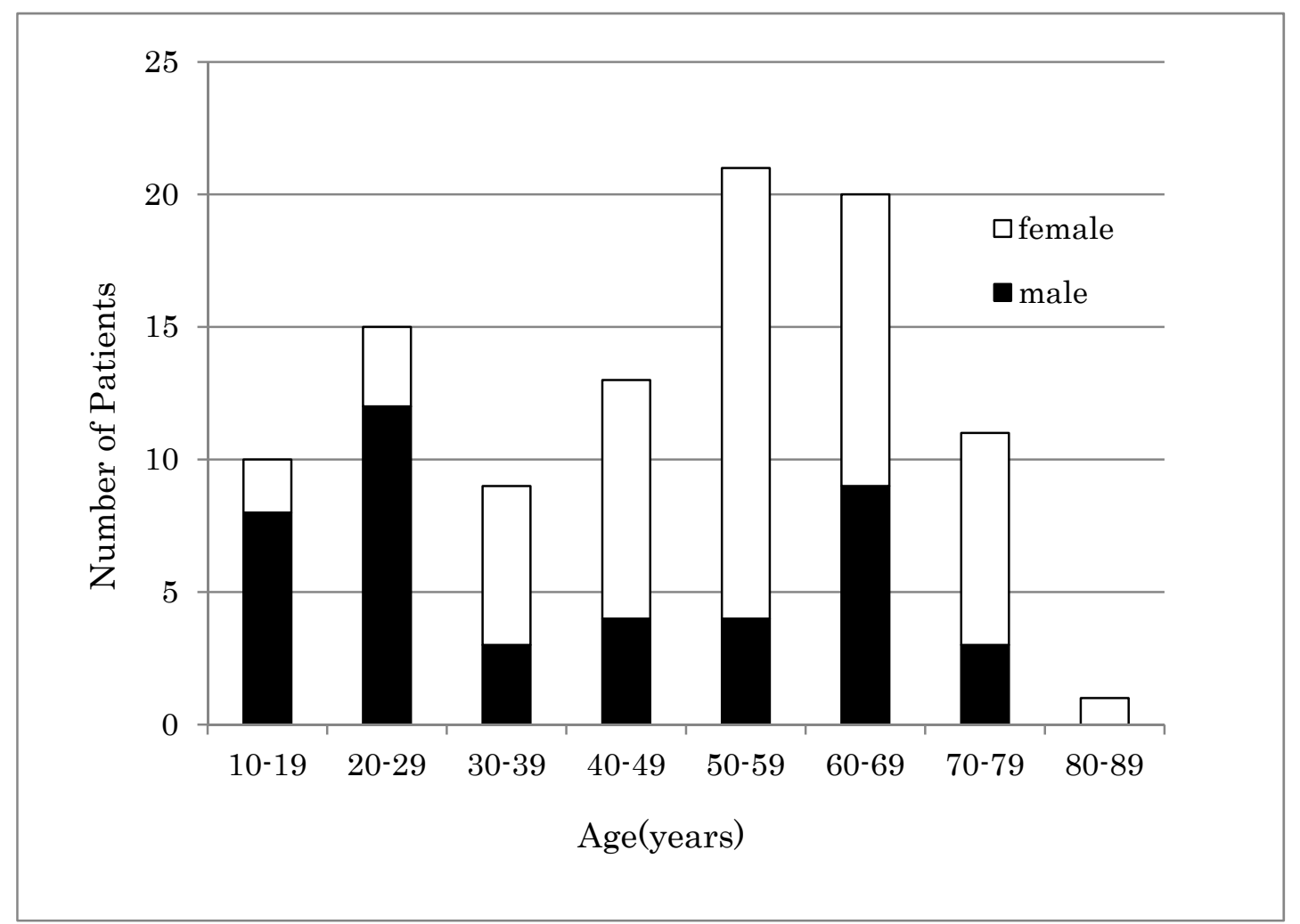

